\title{
Effects of finite pulse length, magnetic field, and gas ionization on ion beam pulse neutralization by background plasma
}

\author{
Igor D. Kaganovich ${ }^{\mathrm{a}, *}$, Adam B. Sefkow ${ }^{\mathrm{a}}$, Edward A. Startsev ${ }^{\mathrm{a}}$, \\ Ronald C. Davidson ${ }^{\mathrm{a}}$, Dale R. Welch ${ }^{\mathrm{b}}$ \\ ${ }^{a}$ Plasma Physics Laboratory, Princeton University, Princeton, NJ, USA \\ ${ }^{\mathrm{b}}$ Voss Scientific, Albuquerque, NM, USA
}

Available online 22 February 2007

\begin{abstract}
This paper presents a survey of the present theoretical understanding of plasma neutralization of intense heavy ion beams. Particular emphasis is placed on determining the degree of charge and current neutralization. We previously developed a reduced analytical model of beam charge and current neutralization for an ion beam pulse propagating in a cold background plasma. The model made use of the conservation of generalized fluid vorticity. The predictions of the analytical model agree very well with numerical simulation results. The model predicts very good charge neutralization during quasi-steady-state propagation, provided the beam pulse duration is much longer than the electron plasma period. In the opposite limit, the beam pulse excites large-amplitude plasma waves. If the beam density is larger than the background plasma density, the plasma waves break, which leads to electron heating. The reduced-fluid description provides an important benchmark for numerical codes and yields useful scaling relations for different beam and plasma parameters. This model has been extended to include the additional effects of a solenoidal magnetic field, gas ionization and the transition regions during beam pulse entry and exit from the plasma. Analytical studies show that a sufficiently large solenoidal magnetic field can increase the degree of current neutralization of the ion beam pulse. However, simulations also show that the self-magnetic field structure of the ion beam pulse propagating through background plasma can be complex and non-stationary. Plasma waves generated by the beam head are greatly modified, and whistler waves propagating ahead of the beam pulse are excited during beam entry into the plasma. Accounting for plasma production by gas ionization yields a larger self-magnetic field of the ion beam compared to the case without ionization, and a wake of the current density and self-magnetic field are generated behind the beam pulse. Beam propagation in a dipole magnetic field configuration and background plasma has also been studied.
\end{abstract}

(C) 2007 Elsevier B.V. All rights reserved.

PACS: 41.75.Ak; 52.59.Fn

Keywords: Beam-plasma interaction; Magnetic field; Plasma

\section{Introduction}

The neutralization of the ion beam space charge and current by a background plasma is an important issue for many applications involving the transport of positive charges in plasma, including heavy ion fusion [1-4], positrons for electron-positron colliders [5], intense laserproduced proton beams for the fast ignition of inertial confinement fusion targets [6], production of charge-

\footnotetext{
*Corresponding author. Tel.: +609243 3277.

E-mail address: ikaganov@pppl.gov (I.D. Kaganovich).
}

compensated intense proton beams in an accelerating ring at currents above the space-charge limit [7], etc.

To neutralize the large repulsive space-charge force of an intense ion beam, the beam pulses can be transported through a background plasma. The plasma electrons can effectively neutralize the ion beam charge, and the background plasma can provide an ideal medium for ion beam transport and focusing. There are many critical parameters for ion beam transport, including beam current, type of ion species, transverse and longitudinal profiles of the beam density, gas density, stripping and ionization crosssections, etc. Because detailed beam and plasma parameter 
values are not always well prescribed, an extensive study is necessary for a wide range of beam and plasma parameters to determine the conditions for optimum beam propagation and focusing [8]. To complement the numerical simulation studies, a number of reduced theoretical models have been developed. Based on well-verified assumptions, reduced models can yield robust analytical and numerical descriptions and provide important scaling laws for the degrees of charge and current neutralization [9-11]. The initial designs for ion beam propagation in the target chamber invoked unneutralized beam transport. However, due to the large space-charge and possible gas and vapor presence in the chamber, neutralized beam transport appears to be a more practical option. An intense ion beam pulse that has considerable ion charge attracts electrons from the ambient plasma background, producing incomplete charge neutralization. The incomplete neutralization results in nonlinear, uncontrollable forces on an ion beam pulse, which inhibit focusing to a small spot size. Therefore, nearly complete charge neutralization of the ion beam pulse appears to be the only practical solution for ballistic focusing of intense ion beam pulses.

This paper presents a survey of the present theoretical understanding of the neutralization of intense heavy ion beams by background plasma. The organization of the paper is as follows: Section 2 discusses disadvantages of the plasma plug scheme for beam neutralization, Section 3 identifies the key plasma parameters for good charge and current neutralization of the ion beam pulse, Section 4 highlights the main results of nonlinear reduced analytical models describing the degree of charge and current neutralization by background plasma, and Sections 5-7 describe the effects of a solenoidal magnetic field, gas ionization, and a dipole magnetic field, respectively, on the self-electric and self-magnetic fields of an ion beam pulse propagating in a background plasma.

\section{Disadvantages of plasma plug scheme for beam neutralization}

Previous studies have explored the option of ion beam pulse neutralization by passing the beam pulse though a layer of plasma or a plasma plug $[3,4,12-14,19]$. The ion beam pulse extracts electrons from the plasma plug and drag electrons along its motion outside the plasma plug region. There are several limitations of this scheme. When the intense beam pulse enters the plasma, the electrons stream into the beam pulse in the strong self-electric and magnetic fields, attempting to drastically reduce the ion beam space charge from unneutralized to a completely neutralized value.

During the entry into the plasma of an intense ion beam pulse with density larger than the background plasma density, a very complex electron response is observed, as shown in Fig. 1. Visualizations (movies) of these processes are available in the supplementary documents to Refs. [11,15]. A current of back-streaming electrons

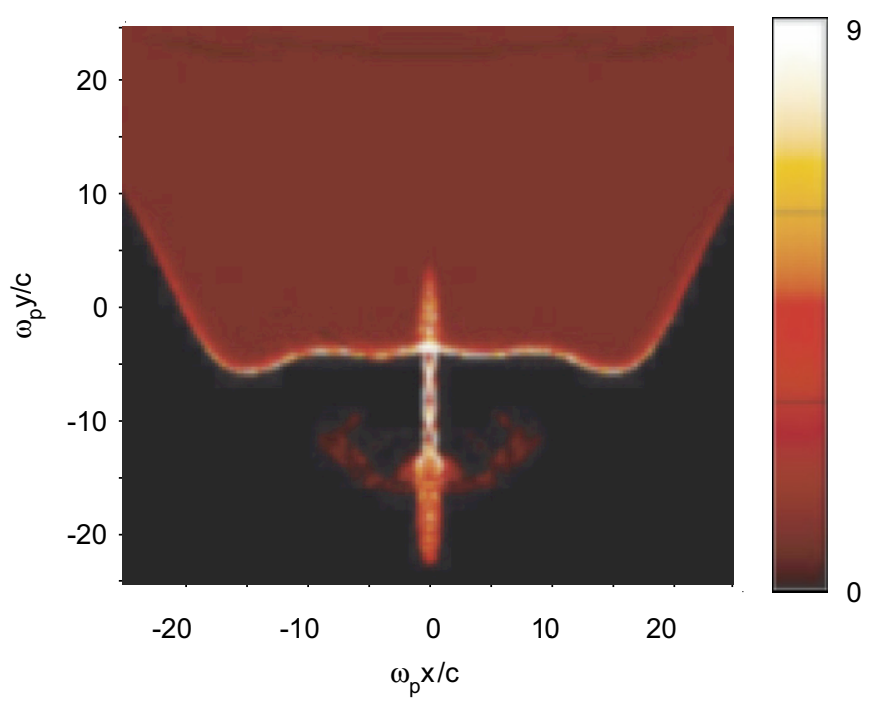

Fig. 1. Neutralization of an ion beam pulse during steady-state propagation of the beam pulse through a cold, uniform, background plasma, calculated using the EDPIC code [9]. The beam propagates in the $y$-direction. Shown in the figure is the color plot of the normalized electron density $\left(n_{\mathrm{e}} / n_{\mathrm{p}}\right)$. The beam velocity is $V_{\mathrm{b}}=0.5 c$, and the beam density is $n_{\mathrm{b}}=5 n_{\mathrm{p}}$. The beam dimensions correspond to $r_{\mathrm{b}}=0.5 c / \omega_{\mathrm{pe}}$ and $\omega_{\mathrm{pe}} \tau_{\mathrm{b}}=120$, where $\tau_{\mathrm{b}}$ is the pulse length.

develops as the unneutralized beam pulse approaches the plasma. This electron current is comparable with the ion beam current and produces a strong self-magnetic field, leading to some hosing effects as shown in Fig. 1. This current flows near the beam axis and results in a nonlinear space-charge force and a substantial beam emittance growth during beam entry into the plasma. At later times than shown in Fig. 1, electron holes are formed inside the beam pulses, which slowly disappear at later times, as shown in the movies in Refs. $[11,15]$.

This process can be violent and complex, as shown in the visualizations in Refs. $[11,15,16]$. The transition region depends on the boundary conditions and on the plasma dimensions. If there is no electron emission from the plasma boundaries, and the plasma's transverse dimension is comparable with the ion beam radius, electron holes form near the plasma boundaries across the beam, because the ion beam pulls electrons in radially from the transverse directions. The electron response time to an external charge perturbation is determined by the electron plasma frequency, $\omega_{\mathrm{pe}}=\left(4 \pi n_{\mathrm{p}} e^{2} / m_{\mathrm{e}}\right)^{1 / 2}$, where $n_{\mathrm{p}}$ is the background plasma density. Therefore, as the ion beam pulse enters the background plasma, the plasma electrons tend to neutralize the ion beam on a time scale of order $\tau_{\mathrm{pe}} \equiv 1 / \omega_{\mathrm{pe}}$. Typically, the ion beam pulse propagation duration through the background plasma is long compared with $\tau_{\mathrm{pe}}$. However, the electron holes exist for a very long time, much longer than the plasma period $\tau_{\mathrm{pe}}$, as one would initially expect [17]. Interestingly, these electron holes move relative to the ion beam pulse with a speed that is a fraction of the beam speed. Thus, the electron holes lag the ion beam pulse and eventually leave the simulation box $[11,16]$. 
After the beam pulse exits the plasma, the beam carries along the electrons, with average electron density and velocity equal to the ion beam's average density and velocity. However, large-amplitude plasma waves are excited in a nonstationary periodic pattern resembling butterfly-wing motion [16]. Due to these transient effects, the beam may undergo transverse emittance growth, which would increase the focal spot size $[4,18]$. Smoother edges to the plasma plug density profile lead to a more gradual neutralization process and, in turn, results in a smaller emittance growth [4].

There are other limitations of this scheme in addition to a deterioration due to transient effects during the beam entry into and exit from the plasma plug. As the beam transversely focuses after passing through the plasma plug, the transverse electron (and ion beam) temperature increases due to the compression and can reach very high values [19]. As a result, the electron Debye length can become comparable with the beam radius, and the degree of charge neutralization reduces considerably. This may result in poor beam focusing. Including gas ionization by the beam ions does not significantly improve the neutralization, mainly because the electrons, which are produced by ionization, are concentrated in the beam path, whereas for effective neutralization of the ion beam pulse, the supply of electrons should be from outside the beam $[4,9,19]$.

Therefore, neutralized ballistic focusing typically requires the presence of background plasma in and around the beam pulse path for good charge neutralization. The presence of cold, "fresh" plasma in the beam path provides the minimum space-charge potential and the best option for neutralized ballistic focusing. Experimental studies of ballistic transverse focusing have confirmed that the best results are achieved when both a plasma plug and a bulk plasma are used for charge neutralization [20].

\section{Key plasma parameters for good charge and current neutralization}

In Refs. [9-11,21], the steady-state propagation of an ion beam pulse through a background plasma has been thoroughly explored. Typically, the beam pulse propagation duration $\tau_{\mathrm{b}}$ through the background plasma is long compared with $\tau_{\mathrm{pe}}$. As a result, after the beam pulse passes through a short transition region, the plasma disturbances are stationary in the beam frame. We have developed reduced nonlinear models, which describe the stationary plasma disturbance (in the beam frame) excited by the intense ion beam pulse. In Refs. $[9,10]$, we have studied the nonlinear quasi-equilibrium properties of an intense, long ion beam pulse propagating through a cold, background plasma, assuming that the beam pulse duration $\tau_{\mathrm{b}}$ is much longer than $\tau_{\mathrm{pe}}$, i.e., $\omega_{\mathrm{pe}} \tau_{\mathrm{b}} \gg 1$. In the study reported in Ref. [11], we extended the previous results to general values of the parameter $\omega_{\mathrm{pe}} \tau_{\mathrm{b}}$. The theoretical predictions agree well with the results of calculations utilizing several particle-incell (PIC) codes; see Refs. [9-11] for additional details.

The model predicts very good charge neutralization during quasi-steady-state propagation, provided the beam is nonrelativistic and the beam pulse duration $\tau_{\mathrm{b}}$ is much longer than the electron plasma period $2 \pi / \omega_{\text {pe }}$, i.e., $\omega_{\mathrm{pe}} \tau_{\mathrm{b}} \gg 2 \pi$. Thus, the degree of charge neutralization depends on the beam pulse duration and plasma density, and is independent of the ion beam current (provided $\left.n_{\mathrm{p}}>n_{\mathrm{b}}\right)$.

However, the degree of ion beam current neutralization depends on both the background plasma density and the ion beam current. The ion beam current can be neutralized by the electron return current. The ion beam charge is neutralized mostly by the action of the electrostatic electric field. In contrast, the electron return current is driven by the inductive electric field generated by the inhomogeneous magnetic flux of the ion beam pulse in the reference frame of the background plasma. Electrons are accelerated in the direction of beam propagation, and thus the electrons tend to neutralize the current as well as the space charge. The inductive electric field penetrates into the plasma over distances of order of the skin depth $c / \omega_{\text {pe }}$. If the beam radius $r_{\mathrm{b}}$ is small compared with the skin depth, $r_{\mathrm{b}}<c / \omega_{\text {pe }}$, then the electron return current is distributed over distances of order $c / \omega_{\mathrm{pe}}$, which is much broader than the ion beam current profile. The magnetic field far away from the beam should decrease to zero. Therefore, the total integrated over a beam cross section current is zero. From Ampere's law, it follows that the electron return current is about $\omega_{\mathrm{pe}} r_{\mathrm{b}} / c$ times less than the ion beam current. Consequently, the ion beam current is neutralized by the electron current, provided the beam radius is large compared with the electron skin depth $c / \omega_{\text {pe }}$, i.e., $r_{\mathrm{b}}>c / \omega_{\mathrm{pe}}$, and is not neutralized in the opposite limit. This condition can be expressed as $[9,10]$

$I_{\mathrm{b}}>4.25\left(\beta n_{\mathrm{b}} / n_{\mathrm{p}}\right) k A$

where $\beta c$ is the directed beam velocity. Fig. 2 shows that good charge neutralization is achieved for $\omega_{\mathrm{pe}} \tau_{\mathrm{b}}=50$.

\section{Nonlinear reduced analytical model for the degree of charge and current neutralization by background plasma}

A theory of the charge and current neutralization of a beam pulse propagating through a background plasma that is based on finding solutions to the equations of motion for a cold electron fluid and Maxwell's equations has been developed $[9,10,23]$. It was shown that Maxwell's equations and the equations of motion of the electrons fluid possess a conservation law for the generalized vorticity $\Omega$, defined by

$\Omega \equiv \nabla \times \mathbf{p}_{\mathrm{e}}-\frac{e}{c} \mathbf{B}$

where $\mathbf{p}_{\mathrm{e}}$ is the electron fluid momentum, and $\mathbf{B}$ is the selfmagnetic field of the beam. Because the generalized 


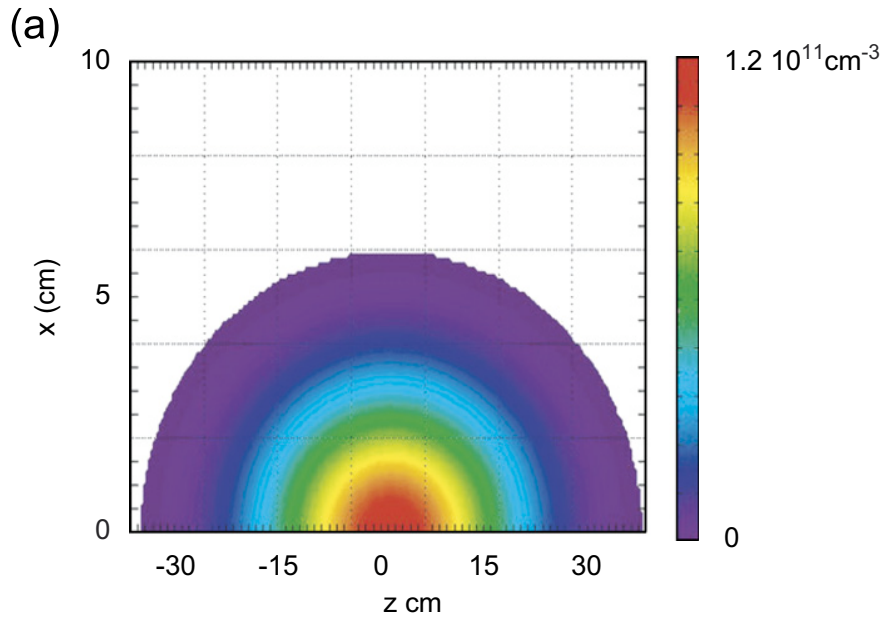

(b)

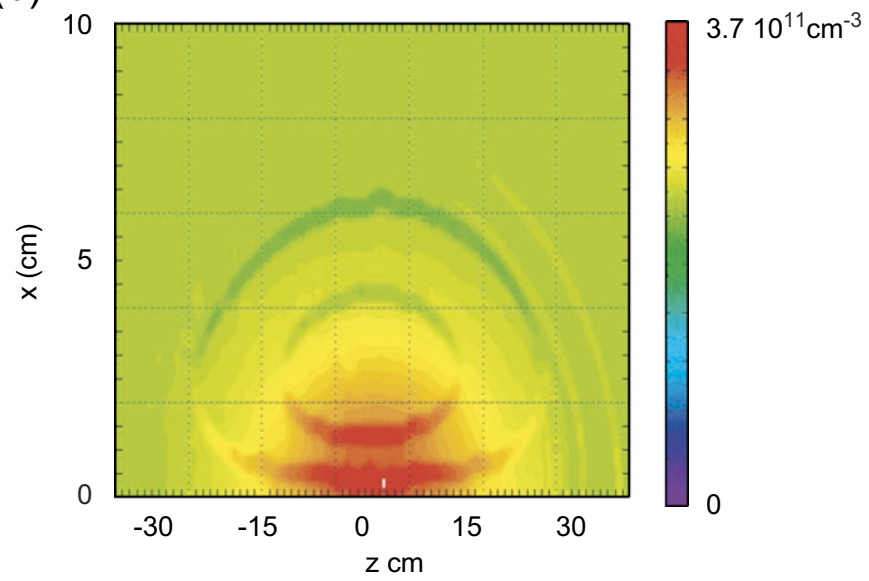

Fig. 2. The electron density perturbation due to the ion beam pulse propagation through a background plasma is calculated in two-dimensional slab geometry using the LSP code [22]. The background plasma density is $n_{\mathrm{p}}=2.410^{11} \mathrm{~cm}^{-3}$. The beam velocity is $V_{\mathrm{b}}=0.33 c$; the beam current density is given by the Gaussian profile $197 \mathrm{~A} / \mathrm{cm} \exp \left(-r^{2} / r_{\mathrm{b}}^{2}-z^{2} /\right.$ $l_{\mathrm{b}}^{2}$ ), which corresponds to the ion beam density $n_{\mathrm{b}}=0.5 n_{\mathrm{p}}$; and the ion beam charge state is $Z_{\mathrm{b}}=1$. The beam dimensions $\left(r_{\mathrm{b}}=3 \mathrm{~cm}\right.$ and $\tau_{\mathrm{b}}=l_{\mathrm{b}} /$ $\left.V_{\mathrm{b}}=1.8 \mathrm{~ns} ; l_{\mathrm{b}}=18 \mathrm{~cm}\right)$ correspond to a beam radius $r_{\mathrm{b}}=2.8 \mathrm{c} / \omega_{\mathrm{pe}}$, and $\tau_{\mathrm{b}} \omega_{\mathrm{pe}}=50$. Shown are color plots of (a) the ion beam density, and (b) the electron density (the transverse structures are an artifact of the code).

vorticity is equal to zero in front of the beam pulse and the generalized vorticity is conserved, it follows from Eq. (2) for long beam pulses with beam half length $l_{\mathrm{b}} \gg r_{\mathrm{b}}$ that

$B=-\frac{c}{e} \frac{\partial p_{\mathrm{e} z}}{\partial r}$

where $B$ is the azimuthal component of self-magnetic field, and axisymmetry is assumed. Note that Eq. (3) expresses the conservation of canonical momentum in the limit of long beam bunches, $l_{\mathrm{b}} \gg r_{\mathrm{b}}$. Eq. (2) is valid even for short beam bunches, where the conservation of canonical momentum is not valid.

The electron return current and self-magnetic field can be obtained from Ampere's law, provided the displacement current can be neglected. Substituting Eq. (3) into
Ampere's law gives $[9,22]$

$-\frac{1}{r} \frac{\partial}{\partial r} r \frac{\partial}{\partial r} V_{\mathrm{e} z}=\frac{4 \pi e}{c m_{\mathrm{e}}}\left(Z_{\mathrm{b}} n_{\mathrm{b}} V_{\mathrm{b} z}-n_{\mathrm{e}} V_{\mathrm{e} z}\right)$.

Here, $Z_{\mathrm{b}}$ is the ion charge of the beam ions. Eq. (4) describes the degree of current neutralization of the beam. For a cylindrical beam, the solution to Eq. (4) for the degree of current neutralization can be approximated to within $5 \%$ accuracy as [10]

$\frac{I_{\text {net }}}{I_{\mathrm{b}}}=\frac{\Lambda\left(f_{\mathrm{p}}\right)}{\left(4 I_{\mathrm{b}}\left(f_{\mathrm{p}}+1\right) / I_{\mathrm{A}}+\Lambda^{2}\left(f_{\mathrm{p}}\right)\right)^{1 / 2}}$

where $\quad \Lambda\left(f_{\mathrm{p}}\right)=2 \sqrt{f_{\mathrm{p}}} /\left(\sqrt{f_{\mathrm{p}}}+\sqrt{f_{\mathrm{p}}+1}\right) . \quad I_{\mathrm{A}} \equiv\left(m_{\mathrm{e}} c^{3} / e\right)$ $\beta_{\mathrm{b}} \approx 17 \beta_{\mathrm{b}} \mathrm{kA}$ is the (nonrelativistic) Alfven-Lawson current for electrons with velocity $\beta_{\mathrm{b}} c$, and

$f_{\mathrm{p}} \equiv n_{\mathrm{p}} /\left(Z_{\mathrm{b}} n_{\mathrm{b}}\right)$

is the normalized background plasma density. The electric field in the radial direction can be calculated from the radial component of the momentum balance equation when the inertia terms can be neglected [23]. This gives

$E_{\mathrm{r}}=\frac{1}{c} V_{\mathrm{e} z} B$.

The radial self-electric field forces electrons into the beam, and beam ions out of the beam. Substituting the magnetic field from Eq. (3) into Eq. (6) gives for the electric field

$E_{\mathrm{r}}=\frac{\partial}{\partial r} m_{\mathrm{e}} V_{\mathrm{e} z}^{2} / 2$

where $V_{\mathrm{e} z}$ has to be calculated from Eq. (4). A comparison of the analytical formulas with the results of the particle-incell code is shown in Fig. 3. As evident from Fig. 3, the agreement is very good.

From Eq. (7), it is evident that the kinetic energy of the electron fluid motion in $z$-direction acts as an effective potential in the radial direction [9]. In the limit of total current neutralization

$$
V_{\mathrm{e} z}=V_{\mathrm{b}} Z_{\mathrm{b}} n_{\mathrm{b}} /\left(n_{\mathrm{p}}+Z_{\mathrm{b}} n_{\mathrm{b}}\right)
$$

and the effective potential is small compared with $m_{\mathrm{e}} V_{\mathrm{b}}^{2} / 2$ by a factor proportional to the square of the ratio of the beam charge density to the plasma density. If the beam current is not fully neutralized, $V_{\mathrm{ez}}$ is even smaller than the value given in Eq. (8). Therefore, increasing the plasma density results in much better neutralization [10]. In Refs. $[14,24]$, it was shown for the case of a plasma plug that the unneutralized electrostatic electric field is reduced after exiting the plasma plug to a value of potential $\phi_{0}$ given approximately by

$\phi_{0}=m_{e} V_{\mathrm{b}}^{2} / 2$.

This potential accelerates the plasma electrons up to the ion beam velocity. Analytical and numerical studies [25,26] show that the potential in Eq. (9) emerges at the end boundary of the neutralization section (plasma plug) as the beam exits this region. The neutralization region may 

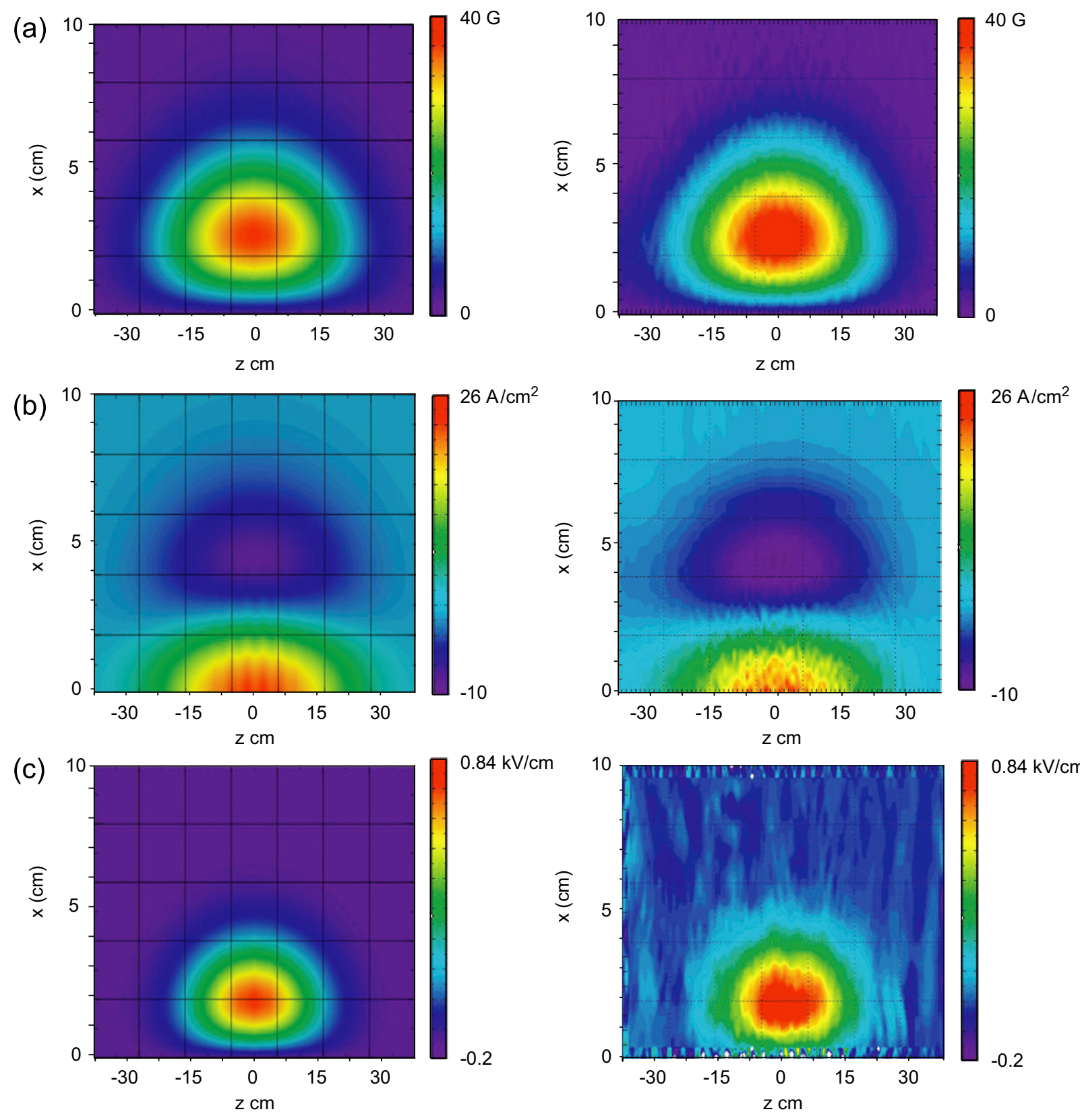

$0.84 \mathrm{kV} / \mathrm{cm}$
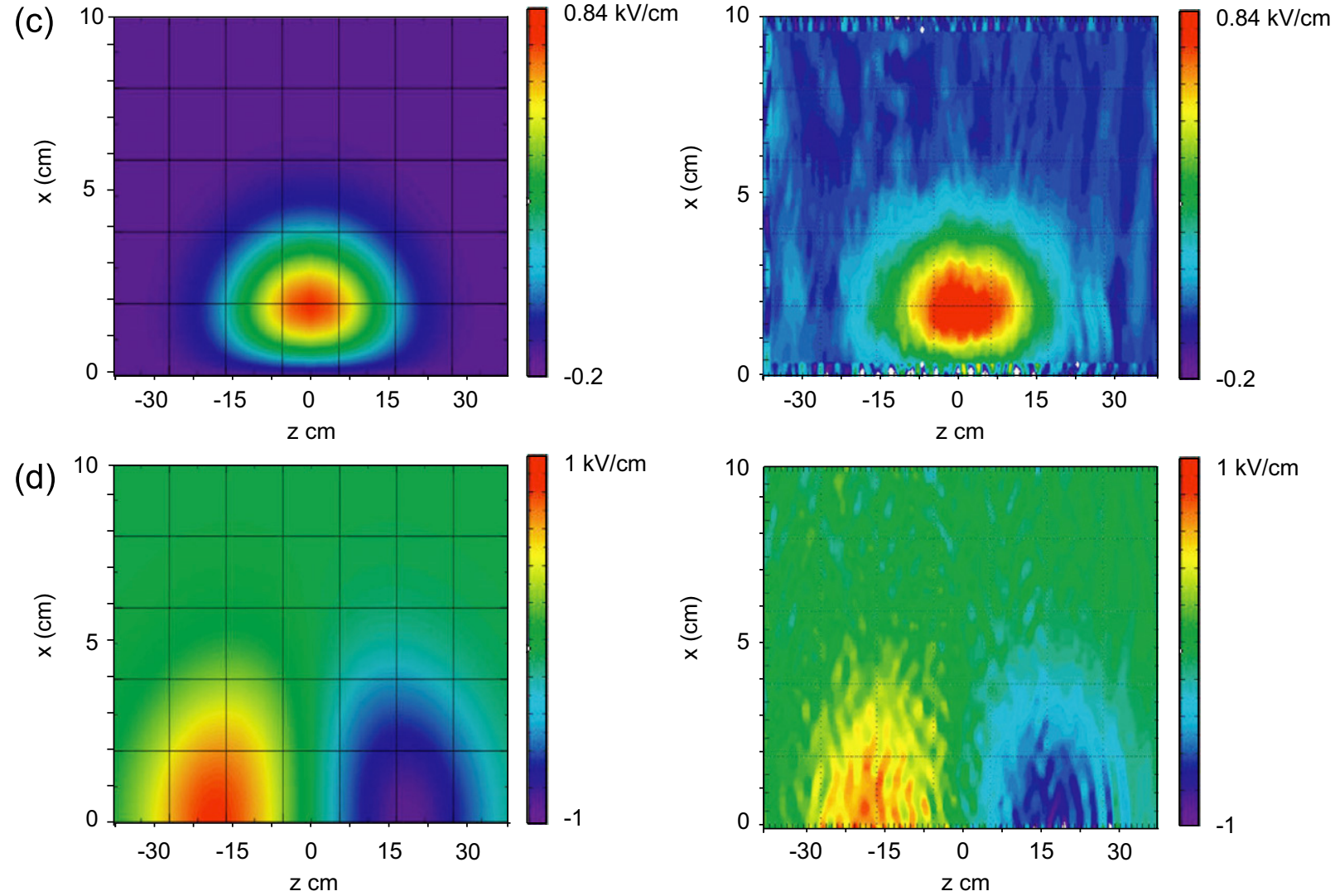

Fig. 3. The self-electric and self-magnetic fields and current density of the ion beam pulse propogating in a background plasma are calculated in twodimensional slab-geometry for the conditions in Fig. 2: (left) reduced analytical model making use of Eqs. (3)-(7), and (right) numerical simulations using the LSP code [22]. Shown are color plots of the radial (c) and longitudinal (d) electric field, current density (b), and the magnetic field component $B_{y}$ (a) generated by the ion beam pulse (top). The streaming factor in electron fluid model in the LSP code is 0.005 . 
consist of an electron-emitting electrode, a biased foil, or a short plasma plug without any background plasma during further propagation of the ion beam. The estimate in Eq. (9) does not pertain to neutralization by the extended background plasma when the beam pulse is immersed inside the plasma, as evident from Eqs. (7) and (8). The potential in Eq. (7) is much less than the estimate in Eq. (9) by a factor proportional to the square of the ratio of the beam charge density to the plasma density.

Note also that the longitudinal electric field is predominantly inductive, and is generated in the plasma due to the time-varying magnetic flux. In the laboratory frame, it is described by the vector potential $\mathbf{A}=A_{z} \mathbf{e}_{z}$, rather than the electrostatic potential $[9,23]$. This has important consequences for beam neutralization. If the theory in Ref. [24] is applied not to the plasma plug but to the propagation in background plasma, it would predict no charge neutralization if the beam density is so small that the unneutralized beam potential satisfies $\phi_{\mathrm{b}} \ll \phi_{0}$, whereas the theory in Ref. [9] predicts neutralization depending on the beam pulse duration. Numerical simulations were performed for an ion beam pulse with $\phi_{\mathrm{b}} / \phi_{0}=\omega_{\mathrm{e}}^{2} r_{\mathrm{b}}^{2} / c^{2} \beta^{2}=0.04$ in Ref. [21], where it was shown that long ion beam pulses with $\omega_{\text {pe }} \tau_{\mathrm{b}} \gg 1$ propagating in volumetric plasma are well neutralized.

In the nonrelativistic limit, the total force acting on the beam ions is [9]

$F_{\mathrm{r}}=m_{\mathrm{e}} e\left(V_{\mathrm{bz}}-V_{\mathrm{e} z}\right) \frac{\partial V_{\mathrm{e} z}}{\partial r}$.

It follows from Eq. (4) that $V_{\mathrm{b} z}>V_{\mathrm{e} z}$ and $\partial V_{\mathrm{e} z} / \partial r<0$, and therefore the force acting on the beam ions in the presence of a dense plasma is always focusing $\left(F_{r}<0\right)$.

The effective self-field perveance can be determined by making use of Eq. (10) for the total force acting on the beam ions at the edge of the beam. The beam perveance $Q_{0}$ in the absence of background plasma is defined by

$Q_{0}=\frac{2 \pi e^{2} Z_{\mathrm{b}}^{2} n_{\mathrm{b}} r_{\mathrm{b}}^{2}}{\gamma_{\mathrm{b}}^{3} M V_{\mathrm{b}}^{2}}$.

In the nonrelativistic regime, and for $r_{\mathrm{b}} \gg \delta_{\mathrm{p}}$ and $f_{\mathrm{p}} \gg 1$, the effective self-field perveance is found [10]:

$Q_{\text {eff }}=-\frac{Z_{\mathrm{b}} m_{\mathrm{e}}}{M} \frac{r_{\mathrm{b}}}{2 \delta_{\mathrm{p}}} \frac{1}{\left(1+f_{\mathrm{p}}\right)}$.

Note that the perveance in Eq. (12) is negative, resulting in a net focusing of the beam ions, but is less in magnitude than the defocusing perveance due to space-charge effects (but can approach it for $r_{\mathrm{b}} \geqslant \delta_{\mathrm{p}}$ and $f_{\mathrm{p}} \sim 1$ ). The perveance in Eq. (12) can be used in the envelope equations to analyze the beam radius dynamics during focusing [8].

The model of plasma neutralization described above is appropriate for quasi-steady-state beam propagation, when variations of the beam pulse parameters are slow compared with the beam pulse duration. In the focal plane, the beam density variation may became fast enough that the assumption of quasi-steady-state fails and the ion beam pulse generates nonstationary waves [27]. In addition, the return current may be subject to the electrostatic twostream instability [18]. Such an instability was observed in 1D simulations in Ref. [18]. However, it was not observed in $2 \mathrm{D}$ simulations, probably due to the shear in electron flow velocity [28].

\section{Effects of solenoidal magnetic field on degree of current and charge neutralization}

We have also studied the influence of an externally applied solenoidal magnetic field on the degree of charge and current neutralization, both analytically and numerically. The applied magnetic field is directed along the ion beam velocity. Analytical studies show that the solenoidal magnetic field begins to influence the radial electron motion when electron cyclotron frequency $\omega_{\mathrm{ce}}=(e B / m c)$, satisfies $\omega_{\mathrm{ce}} \geqslant \omega_{\mathrm{pe}} \beta[21,29]$. If $\omega_{\mathrm{ce}} \ll \omega_{\mathrm{pe}} \beta$, the applied magnetic field does not influence the degree of charge and current neutralization relative to the unmagnetized case. The opposite condition, $\omega_{\mathrm{ce}} \geqslant \omega_{\mathrm{pe}} \beta$, already holds for relatively small magnetic fields: for example, for a $100 \mathrm{MeV}, 1 \mathrm{kA} \mathrm{Ne}^{+}$ion beam $(\beta=0.1)$ and a plasma density of $10^{11} \mathrm{~cm}^{-3}$, the condition $\omega_{\mathrm{ce}}=\omega_{\mathrm{pe}} \beta$ corresponds to a magnetic field of $100 \mathrm{G}$. In the limit $\omega_{\mathrm{ce}} \gg \omega_{\mathrm{pe}} \beta$, the electron return current completely neutralizes the ion beam current. A small unneutralized current is associated with the remnant radial electron transport across the magnetic field and is proportional to $\left(\omega_{\mathrm{ce}} / \omega_{\mathrm{pe}} \beta\right)^{-2}$.

Plasma waves generated by the beam head are greatly modified when $\omega_{\mathrm{ce}}>\omega_{\mathrm{pe}} \beta$, and become whistler waves, in which the electron density perturbations are coupled with electromagnetic perturbations.

Fig. 4 shows the electron density perturbation during beam entry into the uniform background plasma in the presence of a solenoidal magnetic field along the beam propagation direction. Without a solenoidal magnetic field, the wake in the electron density is produced by the ion beam head and lags the ion beam density pulse $[9,11,16]$. Plasma waves form a horizontal stripe pattern in the absence of magnetic field. This structure is greatly modified by the presence of an external magnetic field, as shown in Refs. [21,30]. Moreover, the presence of an external solenoidal magnetic field leads to electron density perturbations propagating away from the beam pulse. During the beam entry into the plasma, electromagnetic perturbations are observed to move ahead of the beam, as shown in Fig. 4. This makes the moving-window computational approach frequently applied in simulations inadequate after the time it takes for the perturbations to reach the front boundary (see Fig. 4), because it is assumed in the moving-window approach that the plasma ahead of the beam pulse is unperturbed. The complete study of this phenomenon is still in progress and shall be reported in Ref. [29] and elsewhere.

The application of an external solenoidal magnetic field leads to the excitation of electromagnetic perturbations at 
(a)

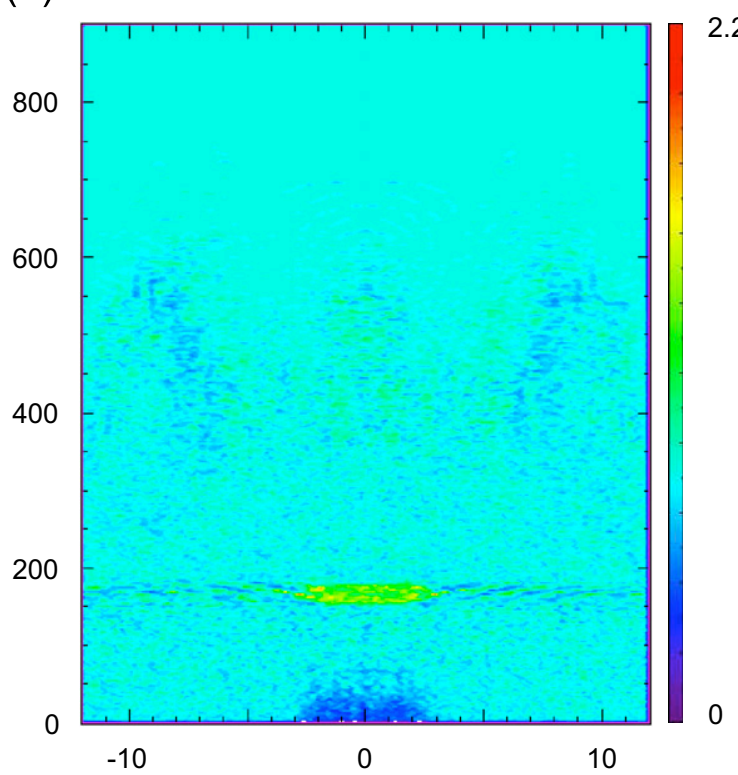

(b)

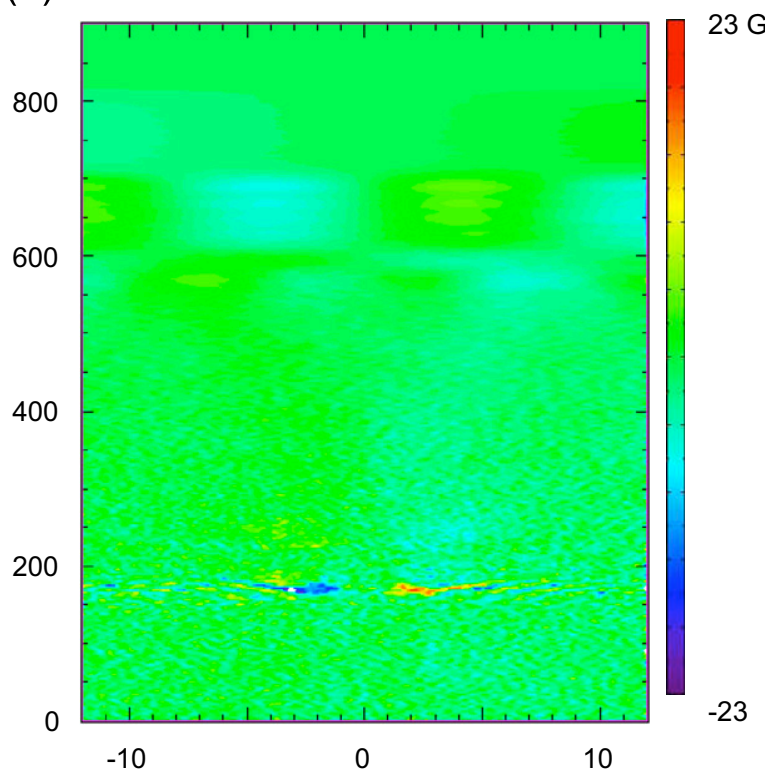

Fig. 4. The charge and current neutralization of the ion beam pulse is calculated in two-dimensional slab geometry using the LSP code [22] for a magnetic field strength corresponding to $\omega_{\mathrm{ce}} / \omega_{\mathrm{p} e}=5.6$. The background plasma density is $n_{\mathrm{p}}=10^{11} \mathrm{~cm}^{-3}$. The beam velocity is $V_{\mathrm{b}}=0.2 c$; the beam current is $1.2 \mathrm{kA}\left(48.0 \mathrm{~A} / \mathrm{cm}^{2}\right)$, which corresponds to the ion beam density $n_{\mathrm{b}}=0.5 n_{\mathrm{p}}$; and the ion beam charge state is $Z_{\mathrm{b}}=1$. The beam dimensions $\left(r_{\mathrm{b}}=2.85 \mathrm{~cm}\right.$ and $\left.\tau_{\mathrm{b}}=1.9 \mathrm{~ns}\right)$ correspond to beam radius $r_{\mathrm{b}}=1.5 c / \omega_{\mathrm{pe}}$, and pulse duration $\tau_{\mathrm{b}} \omega_{\mathrm{pe}}=75$. The solenoidal magnetic field $1014 \mathrm{G}$ corresponds to $\omega_{\mathrm{ce}}=\omega_{\mathrm{pe}}$. Shown are color plots of the electron density (left) and the magnetic field component $B_{z}$ generated by the ion beam pulse (right).

the beam entry into the plasma, which transmit ahead of the beam pulse, as shown in Fig. 4. The application of an external solenoidal magnetic field clearly makes the collective processes in ion beam-plasma interactions considerably more complex and rich in physics content.

\section{Effects of gas ionization on degree of current and charge neutralization}

Gas ionization can considerably affect the degree of current neutralization. For long-beam pulses, the longitudinal canonical momentum is approximately conserved and the electron flow velocity is given by

$v_{\mathrm{e} z}=\frac{e}{m c}\left[A_{z}(z)-A_{z}\left(z_{\mathrm{b}}\right)\right]$

where $A_{z}=\int_{r}^{\infty} B \mathrm{~d} r$ is the vector potential of the selfmagnetic field, and $z_{\mathrm{b}}$ denotes the position where the electron was born in the ionization process. If the electron originated from the background plasma ahead of the beam pulse or in the transverse region outside the beam pulse, where $A_{z}\left(z_{\mathrm{b}}\right)=0$, then the electron flow velocity is proportional to the local value of the vector potential, and we recover Eq. (3). If an electron originates in a region of strong magnetic field, for example, in the beam head, and later moves into a region of weaker magnetic field, then the electron flow velocity is in the direction opposite to the beam velocity. In this case, the current associated with such electrons enhances the beam current does non diminish the beam current, as in the usual case for the return current. From Eq. (13), it is evident that when ionization effects are taken into account the return current becomes nonlocal, i.e., the value of the return current is not only a function of the local plasma density and vector potential, but is also determined by the entire front portion of the beam pulse.

We have performed simulations to determine the importance of ionization effects on the return current. The results are shown in Fig. 5. The beam ionization has an ionization mean-free-path, $l^{i}=45 \mathrm{~cm}$, comparable with the beam length, $2 l_{\mathrm{b}}=36 \mathrm{~cm}$. Therefore, the beam ionizes gas and produces plasma with density comparable with the beam density, as shown in Fig. 5. The gas is located at $x>110 \mathrm{~cm}$ and has a smooth profile from $x=110$ to $140 \mathrm{~cm}$. The plasma density that is produced is given by

$n^{i}(\mathbf{x}, t)=\frac{1}{l^{i}} \int_{z}^{\infty} \mathrm{d} z^{\prime} n_{\mathrm{b}}\left(\mathbf{x}^{\prime}, t\right)$.

For an ion beam pulse with Gaussian profile, the plasma produced by the beam, after the beam pulse passes, has density profile

$n^{i}(r, z)=n_{\mathrm{b} 0} \frac{\sqrt{2 \pi} l_{\mathrm{b}}}{l^{i}} \exp \left(-r^{2} / r_{\mathrm{b}}^{2}\right)$

Due to the presence of the transverse electric field, the electrons produced in the ionization process are pushed into the center of the beam pulse, as evident in Fig. 5(b). As discussed above, the flow velocity of the ionized electrons is less than the velocity of the background electrons. Therefore, the Lorentz force $e v_{\mathrm{e} z} B / c$ cannot compensate for the transverse electric field force $-e E_{r}$, as it does for the case of the flowing electrons from the background plasma. Therefore, the electrons produced in the ionization process are, 
(a)

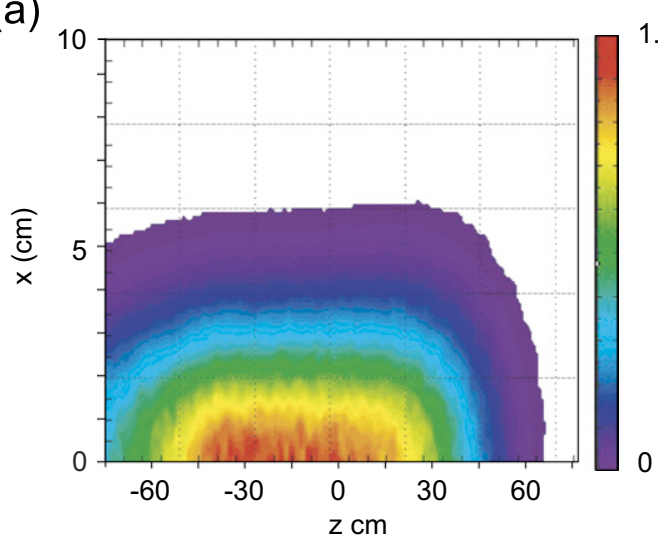

(c)

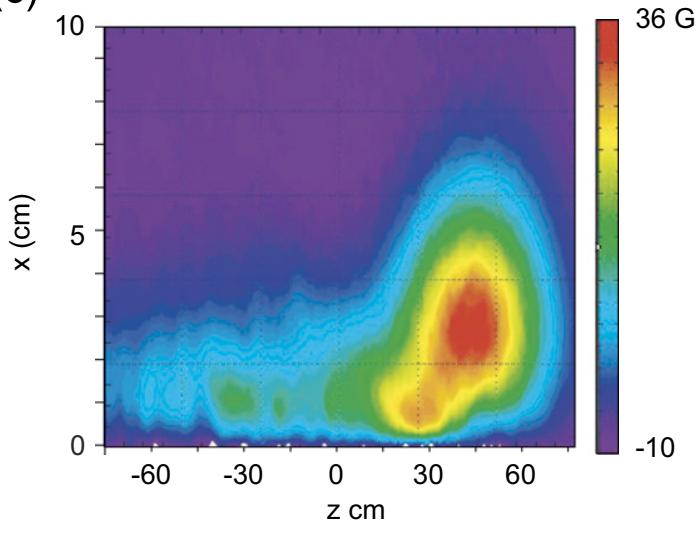

(b)

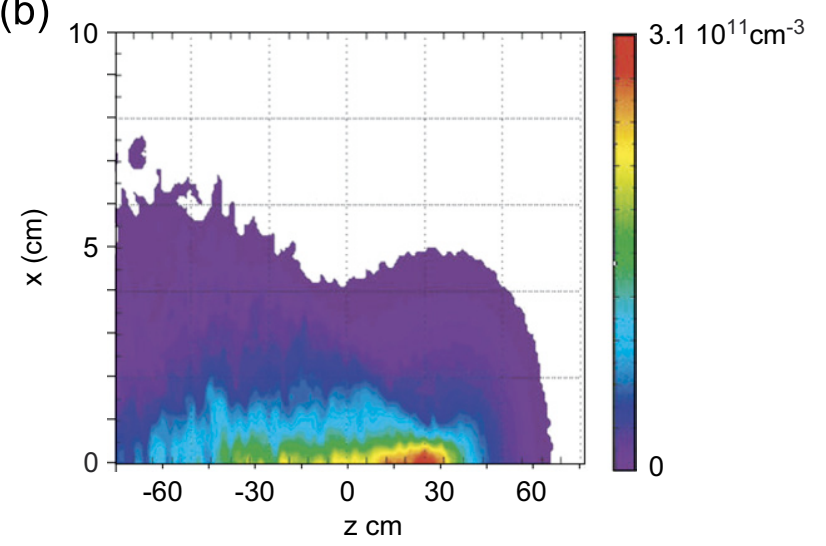

(d)

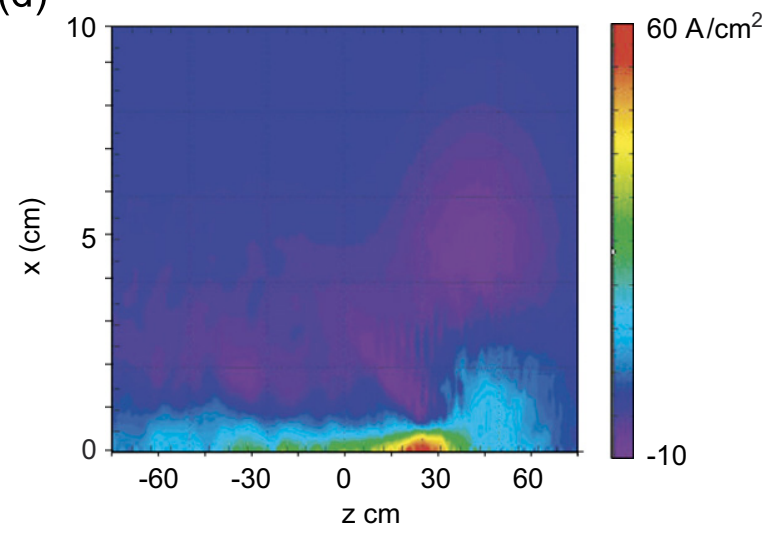

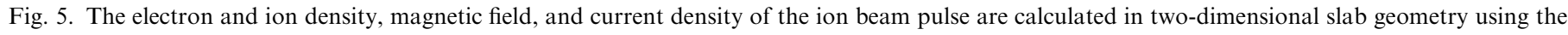

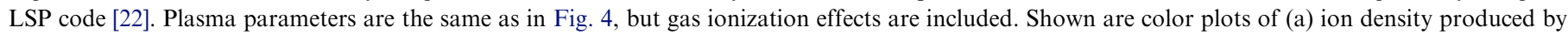

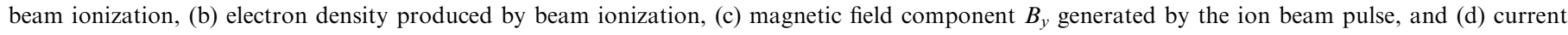
density.

thus, confined in the center of the beam pulse, where they produce an additional current as shown in Fig. 5(d). In a cold, background plasma, the electrons are accelerated by the beam head and decelerated by the beam tail, yielding zero remnant electron energy after interaction with the ion beam pulse. This is not the case for electrons produced by ionization and they are left with some remnant flow velocity in the direction opposite to the beam velocity. As a result, when the beam pulse ends, the electrons produced by ionization generate a long tail in the magnetic field profile, as evident in Fig. 5(c). A detailed discussion of this phenomenon will be presented in a future publication.

\section{Effects of dipole magnetic field on degree of current and charge neutralization}

A dipole magnetic field can be used to deflect the beam. Due to the large ion beam space charge, it is necessary to fill the dipole region with a background plasma to neutralize the beam space charge. The question arises as to whether the plasma can still neutralize the ion beam space-charge density effectively. We performed numerical simulations initially in two-dimensional geometry, and found that beam charge is completely not neutralized. However, to obtain physically correct results, it appears that the simulations have to be carried out in threedimensional geometry. This is because it is necessary to take into account the plasma flows in all directions simultaneously: along the dipole magnetic field, and across the magnetic field, in order to properly take into account all the drifts and flows set up in a dipole magnetic field, when the beam pulse moves in background plasma. In this case, the beam space-charge density is neutralized by the plasma flow along the dipole magnetic field, and the space charge appears to be well-neutralized overall. However, because the electron motion across the magnetic field is greatly reduced by the dipole magnetic field, the current is almost completely unneutralized, as shown in Fig. 6. The unneutralized current generates a time-varying self-magnetic field in the laboratory frame, which in turn produces an inductive electric field $E_{z}$, as shown in Fig. 6(e). The longitudinal electric field $E_{z}$ produces drifts in the $x$ direction and polarizes the plasma, as evident in Fig. 6(f). After the beam exits the dipole region, the current becomes neutralized as shown in Fig. 6(d). However, some complex 
(a)

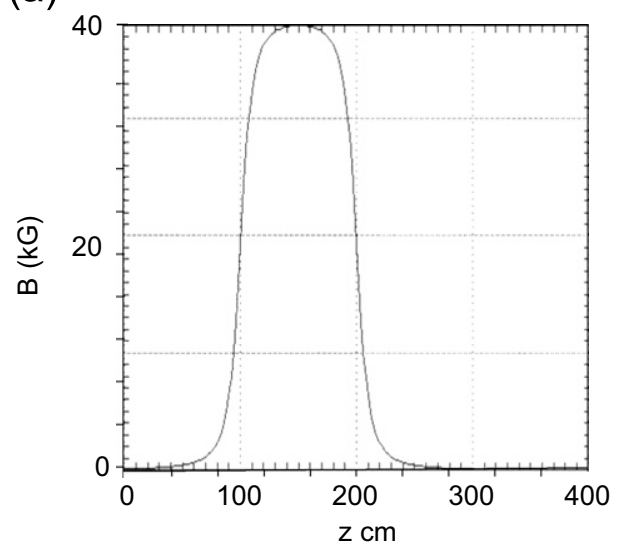

(c)

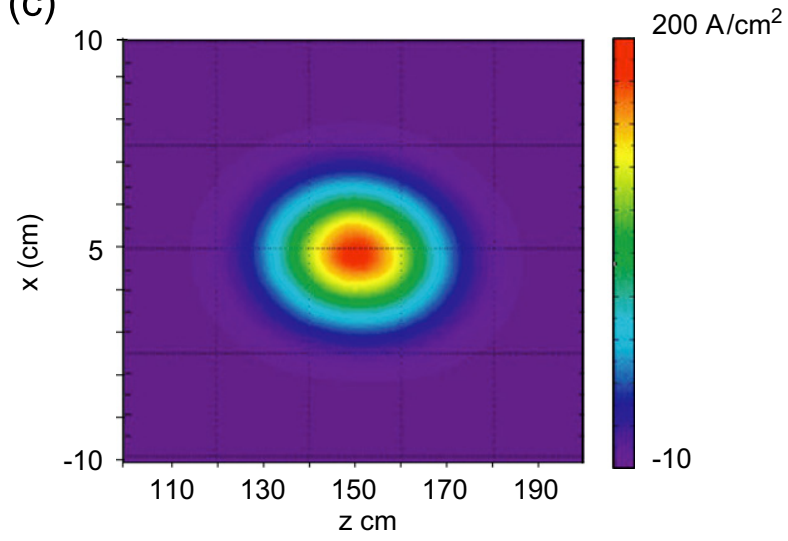

(e)

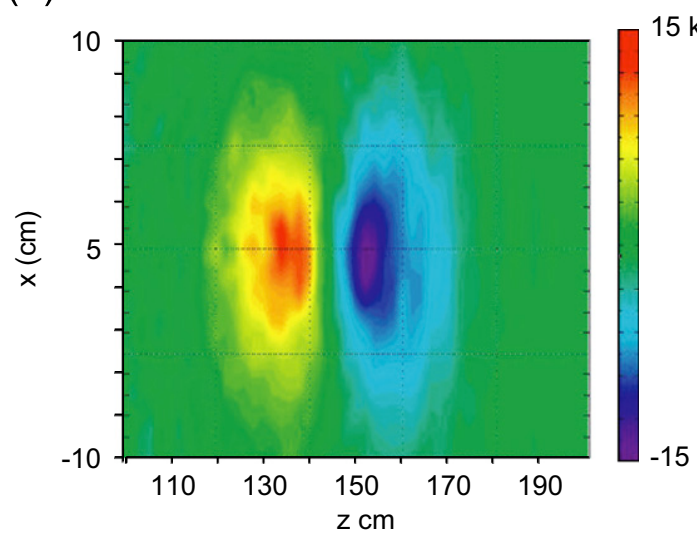

(b)

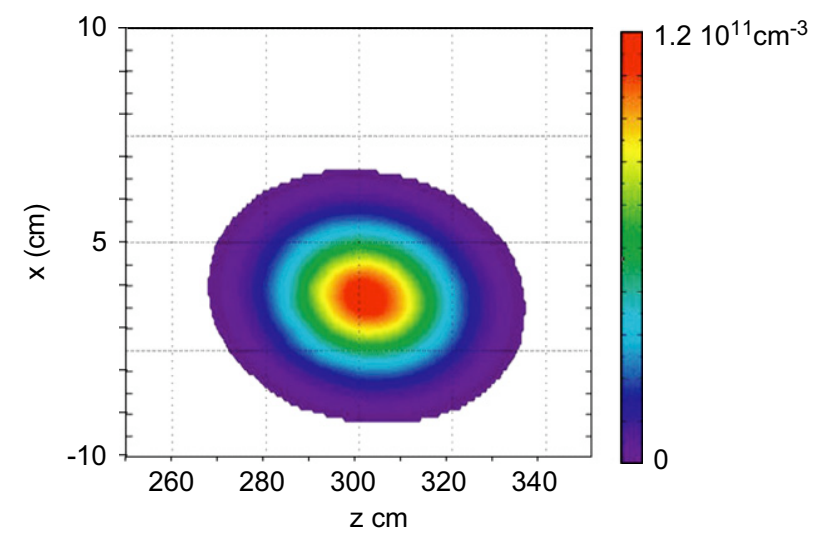

(d)

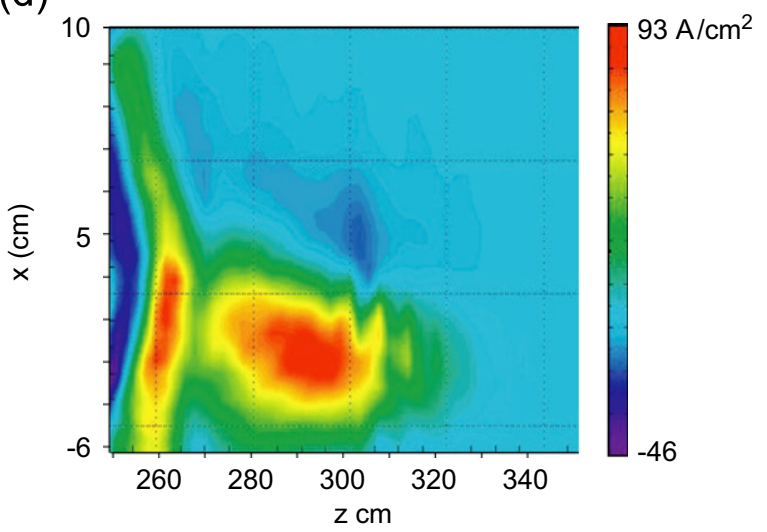

(f)

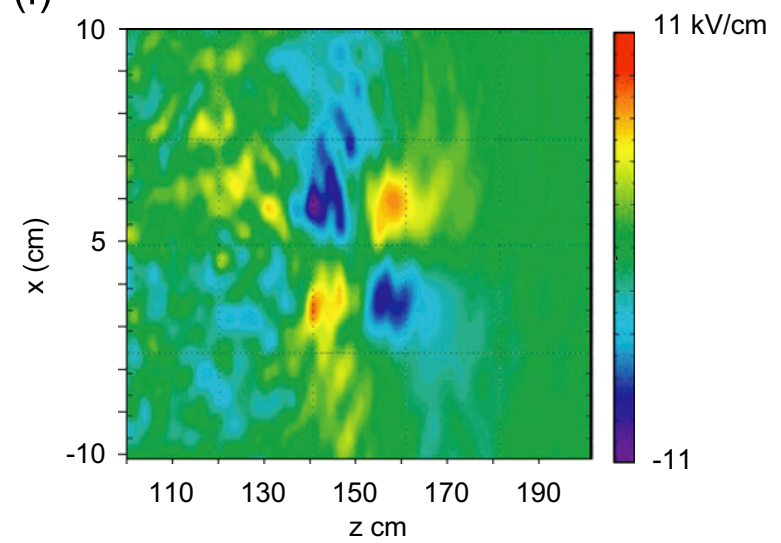

Fig. 6. Beam propagation in a dipole magnetic field. Plots correspond to (a) magnetic field of the dipole, $B_{y}$, (b) beam density in the dipole region, (c) current density in the dipole region, $j_{z}$, (d) current density outside the dipole region, $j_{z}$, (e) longitudinal, inductive electric field, $E_{z}$, and (f) transverse electric field, $E_{x}$. The beam parameters are the same as in Fig. 5.

structures appear at the dipole boundary, as evident from comparing the color plots of the beam density in Fig. 6(b) and the current density in Fig. 6(d).

\section{Conclusions}

We previously developed a reduced analytical model of beam charge and current neutralization for an ion beam pulse propagating in a cold background plasma. The model uses the fluid conservation law for the generalized vorticity.
The predictions of the analytical model agree very well with numerical simulation results. The model predicts very good charge and current neutralization by plasma electrons for intense ion beam pulses. This model has been extended to include two important effects: gas ionization, and applied magnetic field.

Electrons in the background plasma are accelerated by the inductive electric field in the head of the beam pulse and move in the direction of the ion beam velocity. As a consequence, the electrons tend to neutralize the ion beam 
current. If the electrons are generated inside the beam, instead of entering the beam pulse from the front of the beam pulse, e.g., by the ionization of background gas, these electrons do not interact with the strong electromagnetic field in the head of beam pulse, and their flow velocity is smaller. In a cold, background plasma, the electrons are accelerated by the beam head and decelerated by the beam tail, yielding zero remnant electron energy after interaction with the ion beam pulse. For the case of gas ionization, the electrons are produced inside the beam. Therefore, they leave the beam pulse with a remnant flow velocity in the direction opposite to the beam velocity. These electrons generate a long tail in the current and magnetic field profiles behind the beam pulse. The self-electric and magnetic field of an ion beam pulse is modified, when gas ionization is accounted for. This may lead to different focusing of the head of an ion beam pulse compared with the tail of the pulse. An estimate of self-electromagnetic field presented in the paper can be used to quantify this effect.

The application of an external solenoidal magnetic field leads to the excitation of electromagnetic perturbations at the beam entry into the plasma, which are observed to move ahead of the beam pulse. In general, the degree of current neutralization increases and the degree of charge neutralization decreases with applied solenoidal magnetic field strength. The plasma waves excited by the beam head transform into whistler or lower-hybrid waves, and their structure becomes more complex than in the absence of solenoidal magnetic field. In summary, the application of an external solenoidal magnetic field clearly makes the collective in ion beam-plasma interaction processes considerably more complex and rich in physics content. Again, presence of large amplitude lower-hybrid waves may deteriorate the ion beam pulse focusing [27]. Detection of these waves can be used as a diagnostics of beam-plasma interaction.

For beam propagation in a dipole magnetic field applied to deflect the beam, the beam charge density is neutralized by the plasma flow along the dipole magnetic field lines, and the beam space charge appears to be well neutralized. However, because the electron motion across the dipole magnetic field is greatly reduced by the dipole magnetic field, the current is almost completely unneutralized. An estimate of self-electromagnetic field inside the dipole presented in the paper can be used to calculate aberrations by a dipole immersed into the plasma.

\section{Acknowledgment}

This research was supported by the US Department of Energy under the auspices of the Heavy Ion Fusion Science Virtual National Laboratory.

\section{References}

[1] B.G. Logan, et al., Phys. Plasmas 10 (2003) 2063; B.G. Logan, et al., Nucl. Instr. and Meth. A (2007).

[2] S.S. Yu, et al., Fusion Sci. Technol. 44 (2003) 266.

[3] W.M. Sharp, et al., Fusion Sci. Technol. 43 (2003) 393.

[4] W.M. Sharp, et al., Fusion Sci. Technol. 44 (2004) S221.

[5] M.J. Hogan, et al., Phys. Rev. Lett. 90 (2003) 205002.

[6] K. Krushelnick, et al., IEEE Trans. Plasma Sci. 28 (2000) 1184.

[7] G. Dimov, V. Chupriyanov, Particle Accelerators 14 (1984) 155.

[8] J. Barnard, et al., Nucl. Instr. and Meth. A 544 (2005) 243.

[9] I.D. Kaganovich, et al., Phys. Plasmas 8 (2001) 4180; also available at 〈http://w3.pppl.gov/ ikaganov/Publications.htm\#IonBeamPlasma 〉

[10] I.D. Kaganovich, E. Startsev, R.C. Davidson, Laser Particle Beams 20 (2002) 497.

[11] I.D. Kaganovich, E. Startsev, R.C. Davidson, Phys. Plasmas 11 (2004) 3546.

[12] D. Callahan, Fusion Eng. Design 32-33 (1996) 441.

[13] D.R. Welch, et al., Nucl. Instr. and Meth. A 544 (2005) 236.

[14] D.R. Welch, et al., Laser Particle Beams 20 (2002) 621.

[15] High Intensity Particle Beams and Nonneutral Plasma Division, Plasma Physics Laboratory, Princeton University; 〈http://w3.pppl. gov $/ \sim \operatorname{nnp} />$.

[16] I.D. Kaganovich, E.A. Startsev, R.C. Davidson, Phys. Scripta T 107 (2004) 54 〈http://www.pppl.gov/pub_report//2004/PPPL3909-abs.html>.

[17] P. Stroud, Laser Particle Beams 4 (1986) 261.

[18] D.R. Welch, et al., Nucl. Instr. and Meth. A 544 (2005) 236.

[19] A.F. Lifschitz, G. Maynard, J.L. Vay, Nucl. Instr. and Meth. A 544 (2005) 202

[20] P. Roy, S. Yu, et al., Phys. Plasmas 11 (2004) 2890; P. Roy, et al., Phys. Rev. Lett. 95 (2005) 234801; P. Roy, et al., Nucl. Instr. and Meth. A 544 (2005) 225.

[21] I.D. Kaganovich, E. Startsev, R.C. Davidson, D. Welch, Nucl. Instr. and Meth. A 544 (2005) 383.

[22] D.R. Welch, et al., Phys. Plasmas 9 (2002) 2344.

[23] K. Hahn, E.P.J. Lee, Fusion Eng. Design 32-33 (1996) 417.

[24] C. Olson, et al., Fusion Eng. Design 32-33 (1996) 485.

[25] S. Humphreys, T.R. Lockner, J.W. Poukey, J.P. Quintenz, Phys. Rev. Lett. 46 (1981) 995.

[26] R.N. Sudan, App. Phys. Lett. 44 (1984) 958.

[27] A. Sefkow, et al., Nucl. Instr. and Meth. A (2007).

[28] E.A. Startsev, et al., Nucl. Instr. and Meth. A (2007).

[29] I.D. Kaganovich, E. Startsev, R.C. Davidson, Physics of Plasmas (2007), submitted.

[30] I.D. Kaganovich, E. Startsev, R.C. Davidson, IEEE Trans. Plasma Sci. 33 (2005) 556. 